\title{
Treatment of Radiation-Induced Hemorrhagic Duodenitis with Argon Plasma Coagulation
}

Gastrointestinal bleeding from chronic radiation duodenitis is rare. We present a case of radiation-induced hemorrhagic duodenitis successfully treated with argon plasma coagulation (APC) using a two-channel endoscope with a disposable distal attachment. The patient was a 75-year old woman who had advanced-stage hepatocellular carcinoma with metastasis to the lymph nodes of the hilum. She had received palliative treatment with transcatheter arterial embolization of the liver mass and radiation therapy to the hilar lymph nodes. She was referred to our hospital nine month later for radiation-induced hemorrhagic duodenitis with melena. She had received transfusion of 8 units of packed red blood cells over the previous 4 weeks. Empirical treatment with omeprazole had been unsuccessful. Upper gastrointestinal endoscopy showed edematous and friable mucosa with multiple telangiectasias in the bulb and descending part of the duodenum (Figure $\mathbf{1} \mathbf{a}$ ). The vascular lesions were treated by APC (Erbe Medical, Tuebingen, Germany) with a 7-Fr probe at a setting of $50 \mathrm{~W}$ and flow rate of $1.5 \mathrm{l} / \mathrm{min}$ (Figure $1 \mathbf{b}$ ). We used a two-channel endoscope (GIF-2T200; Olympus, Tokyo, Japan) with a disposable distal attachment (Olympus). The procedure was repeated at 2, 4, 6, 8 , and 11 weeks after the first APC treatment, when most of the telangiectasias were eradicated (Figure 1 c). After each APC treatment the patient complained of mild epigastric pain, but no serious complications related to the treatment were observed. Melena stopped after the third APC treatment and no more blood transfusion was required. Her hemoglobin level remained stable until her death 8 month later. APC is a diathermybased, noncontact therapeutic modality with a lower risk of perforation compared with other tissue ablation techniques. APC has been used for the treatment of radiation-induced hemorrhagic proctosigmoiditis and carditis [1-6], however, its use in the treatment of radiation-induced hemorrhagic duodenitis has not been previously reported. We initially used a single-channel endoscope, but the procedure became technically difficult due to the strong spasms and bleeding induced by ablation. The two-channel endoscope allowed flushing and suctioning while the distal attachment helped to maintain a good distance between the endoscope and the mucosa for adequate visualization. This made the procedure easier, even in the presence of spasm.

\section{H. Toyoda', E. Jaramillo², K. Mukai' ${ }^{3}$,}

T. Saito ${ }^{3}$, N. Imai ${ }^{3}$, H. Naota ${ }^{3}$, T. Sase ${ }^{3}$, T. Mizuno ${ }^{3}$, H. Shiku ${ }^{3}$, I. Imoto' ${ }^{1}$, Y. Adachi' ${ }^{1}$

${ }^{1}$ Dep. of Endoscopic Medicine, Mie Univ.

School of Medicine, Tsu, Japan

2 Dep. of Gastroenterology and Hepatology, Karolinska Hospital, Stockholm, Sweden ${ }^{3}$ Second Dep. of Internal Medicine, Mie Univ. School of Medicine, Tsu, Japan.

\section{References}

${ }^{1}$ Silva RA, Correia AJ, Dias LM et al. Argon plasma coagulation therapy for hemorrhagic radiation proctosigmoiditis. Gastrointest Endosc 1999; 50: 221 - 224

2 Fantin AC, Binek J, Suter WR et al. Argon beam coagulation for treatment of symptomatic radiation-induced proctitis. Gastrointest Endosc 1999; 49: 515-518

${ }^{3}$ Tam W, Moore J, Schoeman M. Treatment of radiation proctitis with argon plasma coagulation. Endoscopy 2000; 32: 667-672

${ }^{4}$ Kaassis M, Oberti E, Burtin P et al. Argon plasma coagulation for the treatment of hemorrhagic radiation proctitis. Endoscopy $2000 ; 32: 673-676$

${ }^{5}$ MorrowJB, Dumot JA, Vargo JJ III. Radiationinduced hemorrhagic carditis treated with argon plasma coagulator. Gastrointest Endosc 2000; 51:498-499

${ }^{6}$ Canard JM, Védrenne B. Clinical application of argon plasma coagulation in gastrointestinal endoscopy: Has the time come to replace the laser? Endoscopy 2001; 33: $353-357$

\section{Corresponding Author}

\section{H. Toyoda, M.D.}

Department of Endoscopic Medicine, Mie University School of Medicine, 2-174 Edobashi, Tsu, Mie Prefecture 514-8507, Japan Fax: + 81-59-231-5200

E-mail: toyoda-t@ztv.ne.jp
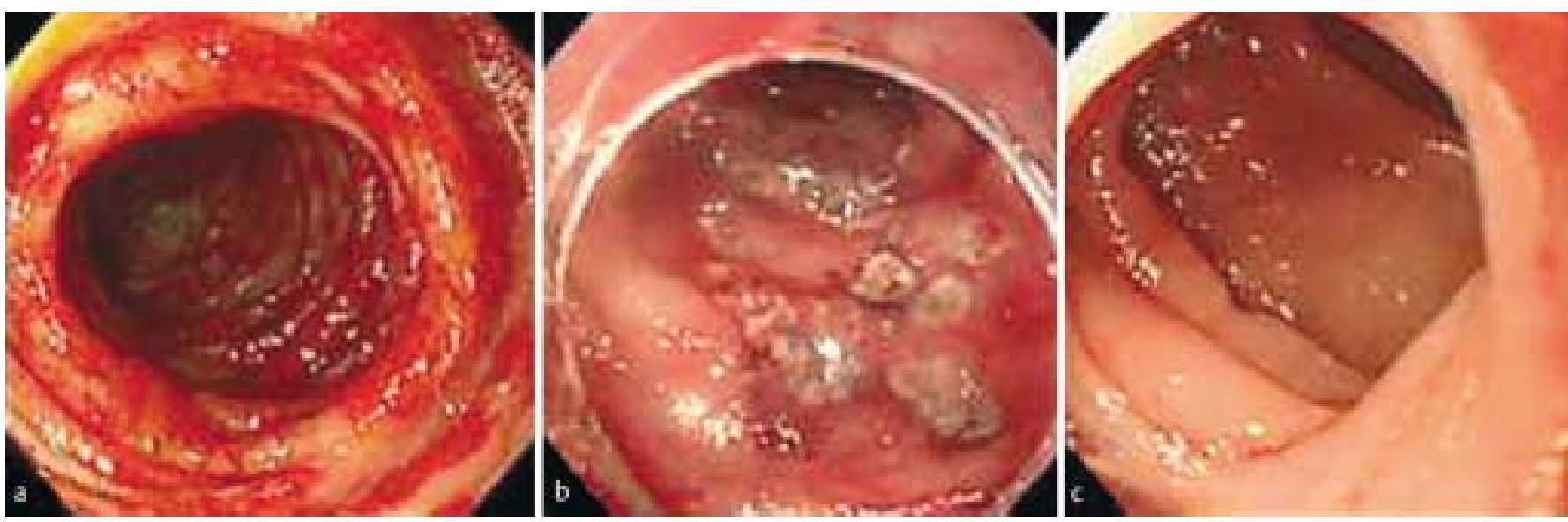

Figure 1 a Endoscopic view of radiation-induced hemorrhagic duodenitis prior to argon plasma coagulation (APC) treatment. Friable mucosa is seen with multiple telangiectasias confined to the bulb and the descending part of the duodenum. $\mathbf{b}$ Areas of superficial coagulation just after the first APC treatment using a two-channel endoscope with a disposable distal attachment. c Endoscopic view after the sixth APC treatment. Most of the telangiectasias have been eradicated. 